\title{
An Environment and Algorithm for FMS Controller Testing ${ }^{\dagger}$
}

\author{
Z. Deng \\ Narvik Institute of Technology \\ Teknologiveien 10, 8501 Narvik, Norway \\ Tel: 47-769-22181 Fax: 47-769-44866 \\ E-mail: Ziqiong.Deng@hin.no \\ Z. Bi and Y. Zhu \\ Division \#503 \\ Nanjing University of Science and Technology \\ Nanjing 210094, P. R. China \\ Tel: 86-25-4315615 Fax: 86-25-4431622
}

\begin{abstract}
The authors have developed a physical environment for testing controllers of Flexible Manufacturing Systems (FMS). In this paper, firstly, the testing mechanism is discussed. Secondly, the architecture of the testing environment is illustrated. Finally, an algorithm based on a model built for testing the correctness of the series of control commands from a tested FMS controller is given. A modelling methodology called Structured Macro Petri Net (SMPN) conceived by authors is described briefly.
\end{abstract}

\section{Keywords}

Software engineering, flexible manufacturing system (FMS), control engineering, software testing, system modelling, Petri net (PN), structured macro Petri net (SMPN)

\footnotetext{
${ }^{\dagger}$ This work is supported with the pre-research foundation by Science and Industry Committee of China.
} 


\section{INTRODUCTION}

For many manufacturers of consumer goods, flexible manufacturing and assembly is the only way in which they can efficiently compete in the market-place with a range of product variants. This is because clients are increasingly looking for products tailored to their own needs rather than mass-produced products (Rembold, 1993). Therefore, for producing such product variants, flexible manufacturing systems (FMS) are required.

Flexible manufacturing systems typically consist of (Deng, 1989):

- several manufacturing equipment (machines) such as CNC machining centres, $\mathrm{CNC}$ measuring machines, washing machines, etc.;

- part transport and handling equipment such as automatic guided vehicle (AGV) together with part loading and unloading stations, central part buffers, and local part buffers dedicated to an individual manufacturing machine to carry out efficient part-flow (or called job-flow) tasks within the FMS system;

- tool transport and exchange equipment such as movable robots together with tool loading and unloading stations, central tool base, and local tool magazines for each manufacturing machine to carry out efficient tool-flow within the FMS system.

All actions that happen among the part-flow procedure or the tool-flow procedure in an FMS are controlled by the FMS controller. In other words, the FMS controller makes a series of decisions and issues a series of commands to control the part-flow and/or the tool-flow in a series of time moments.

Since the early 1980s, there have been more and more FMSs installed in enterprises, and a number of FMS controllers developed by various developers. It seems that there exists a growing tendency of installing FMSs and developing various controllers for those FMSs.

As is well known, developing an FMS controller involves a complicated task in hardware and software development. Especially in the software development, if many bugs exist in the package, probably it may cause a disastrous result while the FMS is running. Therefore, the authors have launched a project since 1991 to develop a testing environment for testing the FMS controllers which may be developed by various developers. In other words, the authors wanted to create a testing centre where any developer or client of an FMS controller can ask the testing centre for the testing and/or debugging of his/her FMS controller. Meanwhile, if someone is willing to develop an FMS controller before his/her physical FMS system installation, he/she may also make use of the facilities of this testing centre to develop his/her FMS controller. It also means that we have to create a virtual equipment environment where the FMS configuration composed of CNC machines, part-flow sub-system, tool-flow subsystem, etc., can be configured for following purposes (Deng, 1995):

- receiving the sequence of control commands from an FMS controller being tested;

- executing the control commands;

- replying with a normal message to inform the FMS controller that a control command is executed properly or;

- replying with a abnormal message in random mode to inform the FMS controller that certain malfunction is happening in the facilities. 
After years' work, the first stage of the testing environment is accomplished. In what follows in this paper, we will describe: (1) the mechanism of FMS controller testing, (2) the architecture of the testing environment, (3) the model and algorithm for testing real-time control function of a tested FMS controller, and (4) macro structurisation of places and resources.

\section{MECHANISM OF FMS CONTROLLER TESTING}

Generally, software production broadly follows the phases: requirements, specifications, design, implementation, integration, maintenance, and finally, retirement (Schach, 1990). After finishing the development of each of the first three phases, verification is required. After each of both the implementation phase and the integration phase, testing is required to assure the quality of that phase's solution. During the implementation phase's testing, the modules are tested. During the integration phase's testing, there are three types of testing required, namely integration testing, product testing, and acceptance testing (Schach, 1990). The purpose of integration testing is to check that the modules are combined together correctly to achieve a software product that satisfies its specifications. When the integration testing has been completed, product testing is performed. The functionality of the product as a whole is checked against the specifications. The final aspect is acceptance testing. Here the client enter the picture. The software is delivered to the client, who tests the software.

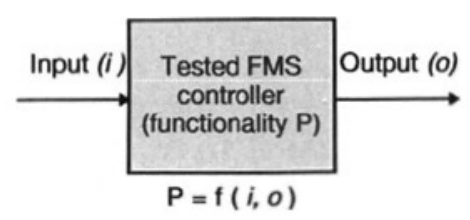

Figure 1 Black-box testing for an FMS controller.

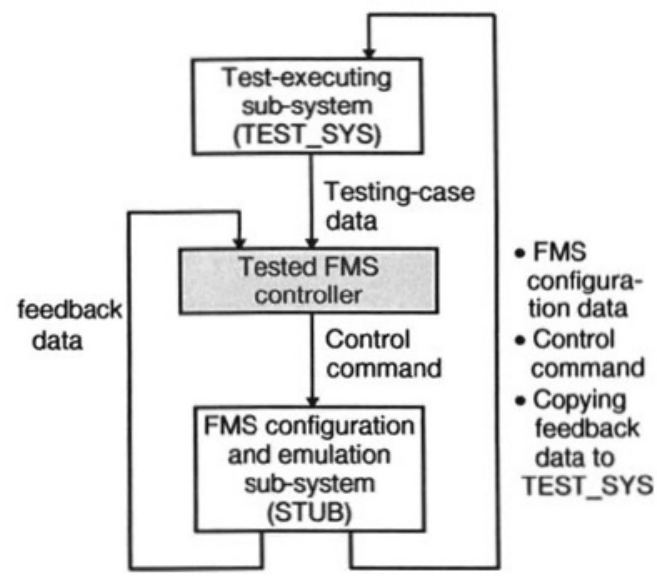

Figure 2 Testing principle of FMS controller's testing.

As mentioned above in the introduction, our desire is to create a testing environment where developers of various FMS controllers can ask the testing environment for the testing of his/her FMS controller. Because a wide variety of FMS controllers are developed outside the testing environment, and the developments are carried out by various groups other than the 
group working in the testing environment, it is not usually possible for the group who works in testing environment to understand the internal software structure of the various tested FMS controllers. That is to say, our work is only involved in product testing and/or acceptance testing if a client wants to use the environment for his/her acceptance testing. Therefore, the mechanism conceived for FMS controllers' testing in our environment is limitedly to the testing of functionality which is specified in the specifications. In other words, the testing environment copes with a tested FMS controller as a black-box as shown in Figure 1.

Considering the relationship between the tested FMS controller and the testing system in our environment, the testing principle can be expressed and depicted as shown in Figure 2 where the testing system is composed of:

- a test-executing sub-system (or called TEST_SYS for brevity);

- an FMS configuration and emulation sub-system (or called STUB for brevity).

Initially, one should make use of the STUB (see lower part of Figure 2) to configure the FMS facilities, part-flow system, and tool-flow system which will be controlled by the tested FMS controller. The FMS configuration data created is then transferred to the TEST_SYS where the FMS configuration data is stored in the database and is ready for use by the TEST_SYS. According to both the functional specifications of the tested FMS controller and the stored FMS configuration data, the TEST_SYS creates various testing-case data (see upper part of Figure 2) to drive the tested FMS controller.

The content of the testing-case data includes the production data or, say, the job assignment which consists of the sets of data included in Tables 1 through 4.

Table 1 Job assignment

\begin{tabular}{c|c|c|c|c|c|c}
\hline No. of job & $\begin{array}{c}\text { Priority of } \\
\text { job }\end{array}$ & Due date & $\begin{array}{c}\text { Type of } \\
\text { part }\end{array}$ & $\begin{array}{c}\text { No. of } \\
\text { blank }\end{array}$ & $\begin{array}{c}\text { Batch } \\
\text { quantity }\end{array}$ & $\begin{array}{c}\text { No. of } \\
\text { process plan }\end{array}$ \\
\hline & & & & & &
\end{tabular}

Table 2 Process plan

\begin{tabular}{c|c|c|c|c|c}
\hline $\begin{array}{c}\text { No. of } \\
\text { process plan }\end{array}$ & $\begin{array}{c}\text { No. of } \\
\text { operation }\end{array}$ & $\begin{array}{c}\text { No. of } \\
\text { equipment }\end{array}$ & $\begin{array}{c}\text { Alternative } \\
\text { equipment }\end{array}$ & Priority & Sequence \\
\hline & & & & &
\end{tabular}

Table 3 Operations

\begin{tabular}{c|c|c}
\hline No. of operation & No. of NC program & Duration of operation \\
\hline & &
\end{tabular}

Table 4 Tool requirement

\begin{tabular}{l|l|l|l}
\hline No. of NC program & No. of tool & Type of tool & Tool life \\
\hline & & &
\end{tabular}


The FMS controller being tested starts to issue its first control command to the STUB according to the testing-case data. Meanwhile, the STUB emulates the execution of the control command, copies the control command to the TEST_SYS, transmits to the tested FMS controller either a normal feedback data, or an abnormal feedback data, and copies as well the feedback data to the TEST_SYS. From the second command on, the FMS controller issues its control commands according to not only the testing-case data, but also the feedback data.

\section{ARCHITECTURE OF TESTING ENVIRONMENT}

Creation of a testing environment involves architectural consideration concerning both the hardware environment and the software environment. The architectural consideration for the hardware environment should address the requirement for serving various sources of FMS controllers which may reside on various types of computers such as Sun workstations, VAX workstations, SGI workstations, HP workstations, and PC computers. Therefore, we have used the hardware configuration as shown in Figure 3.

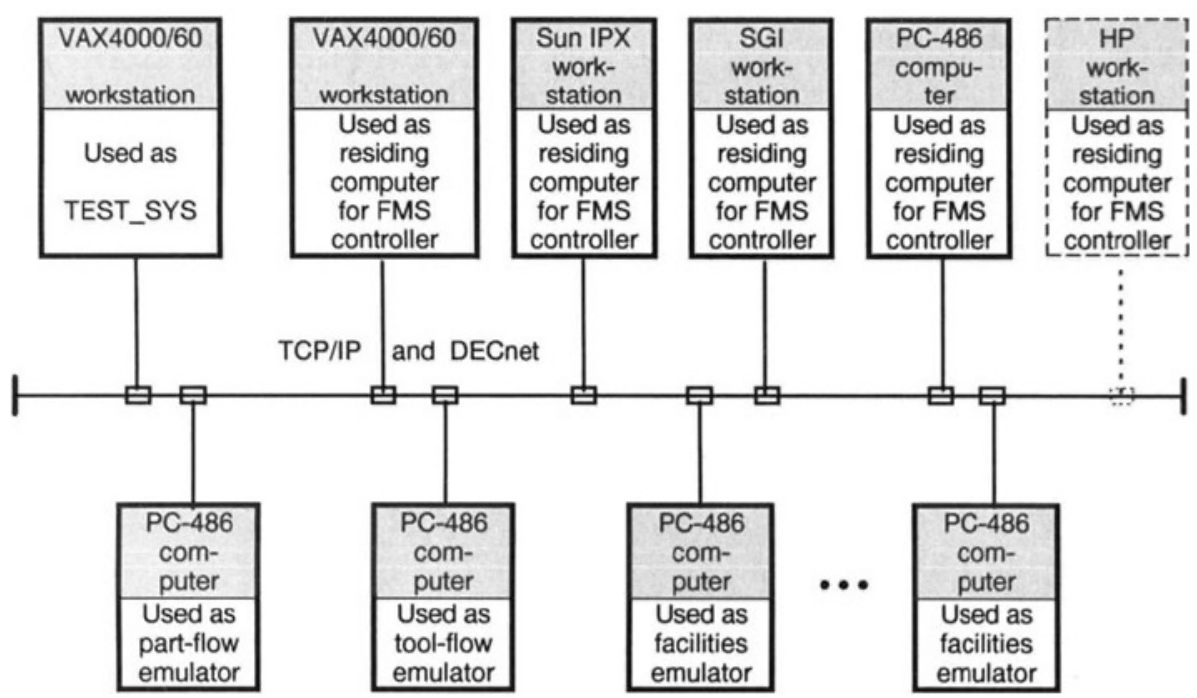

Figure 3 Hardware configuration of testing environment for FMS controllers

The testing-executing sub-system (TEST_SYS) shown in Figure 2 had been installed on one of the VAX 4000/60 workstations as shown in the upper left comer of Figure 3. The STUB had been installed on several PC-486 computers as shown in the lower part of Figure 3. To serve as the residing computers for various sources of FMS controllers, one Sun IPX workstation, one SGI workstation, one PC-486 computer, together with one other VAX $4000 / 60$ workstation had been installed. One HP workstation is planned to be installed soon. 
The architectural considerations for the software environment had led us to adopt the NAS (Network Application Support) platform from Digital Equipment Co. as shown in Figures 4 and 5 (Digital Equipment Co., 1990).

The NAS platform can be used to support a compatible network of multivendor products. As shown in Figure 4, it provides compatibility among different operating systems such as UNIX, VMS, OS/2, MS-DOS, and etc.. As well, it provides compatibility among different databases such as RDB, Ingres, SyBASE, and Oracle. Regarding NAS, we find that it is suitable for meeting our objectives for testing various types of FMS controllers which may be required to work on different computers and databases.

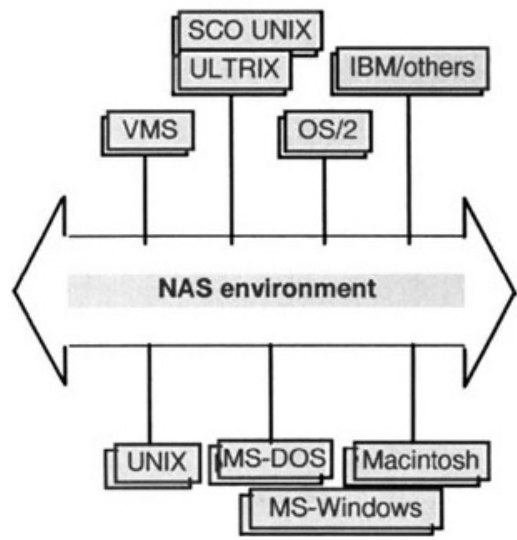

Figure 4 NAS environment for multivendor support

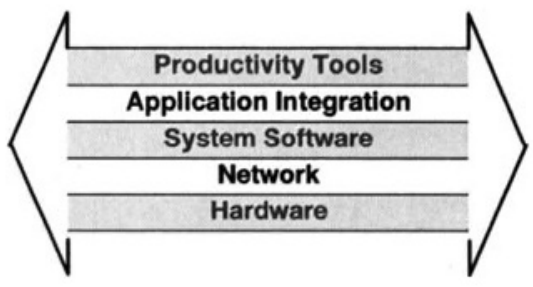

Figure 5 NAS environment for layers

\section{ALGORITHM FOR TESTING REAL-TIME CONTROL FUNCTION OF A TESTED FMS CONTROLLER}

As shown in Figure 2, the TEST_SYS has to

- create a complete set of testing-case data;

- check the correctness of the series of control commands both in normal and in abnormal situations;

and the STUB has to

- emulate the FMS operation;

- generate either normal feedback data, or abnormal feedback data. 
Obviously, we have to conceive algorithms for realising each of the above tasks. In this paper, we only introduce an algorithm for correctness checking of control commands. Other algorithms can be referred to Huang (1995).

To check the correctness of the series of control commands, at first there is a need to create an analytical behaviour model for the TEST_SYS. This model should

- be independent of models which work in tested FMS controllers;

- exactly express the dynamic behaviour of FMS systems which are controlled by tested FMS controllers;

- be as simple as possible for easing the modelling and/or the model extension when needed.

We had conceived a modelling methodology for the TEST_SYS based on Petri-net (PN) theory and methodology (Peterson, 1981). As is well known, PN methodology makes use of places and transitions to graphically build a model in which tokens flow. If we consider the TEST_SYS model where attributes of tokens are related to concrete resources such as jobs (or say, parts), tools, manufacturing machines, etc., as one probably does normally, then here we may encounter the difficulty of unacceptable scale and complexity of the model built with the quantity of resources increasing.

To avoid such problems, we find that there exists a limited number of basic event-types which may happen in an FMS system as follows:

- in job-flow: a job is loading to or unloading from the FMS;

a pallet together with job is transported from one machine to another machine;

a pallet together with job is transported from a buffer to a workstation; a pallet together with job is transported from a workstation to a buffer; a machining operation is started or finished;

- in tool-flow: a tool is loading to or unloading from the FMS;

a tool is transported between tool loading/unloading station and a local tool magazine;

a tool is transported between tool loading/unloading station and central tool magazine;

a tool is transported between a local tool magazine and central tool magazine;

a tool is transported between two of local tool magazines.

Therefore, we firstly structure the places for the TEST_SYS's model, then define the structure of transitions. That is to say, we firstly defined a modelling methodology called SMPN (structured macro Petri-net) methodology with a procedure as follows:

- Resources are classified according to features of their dynamic attributes;

- The sets of places and transitions are structured abstractly to reflect the basic events and related system statuses;

- The global structure of the whole model is then built based on the cause-effect relationship of those basic events. 
Then, using the above modelling methodology, a SMPN model for our testing system was built. In this paper, we only introduce the sub-model for job-flow as shown in Figure 6 . The meanings of various acronyms of both places and transitions in the Figure are explained as follows:

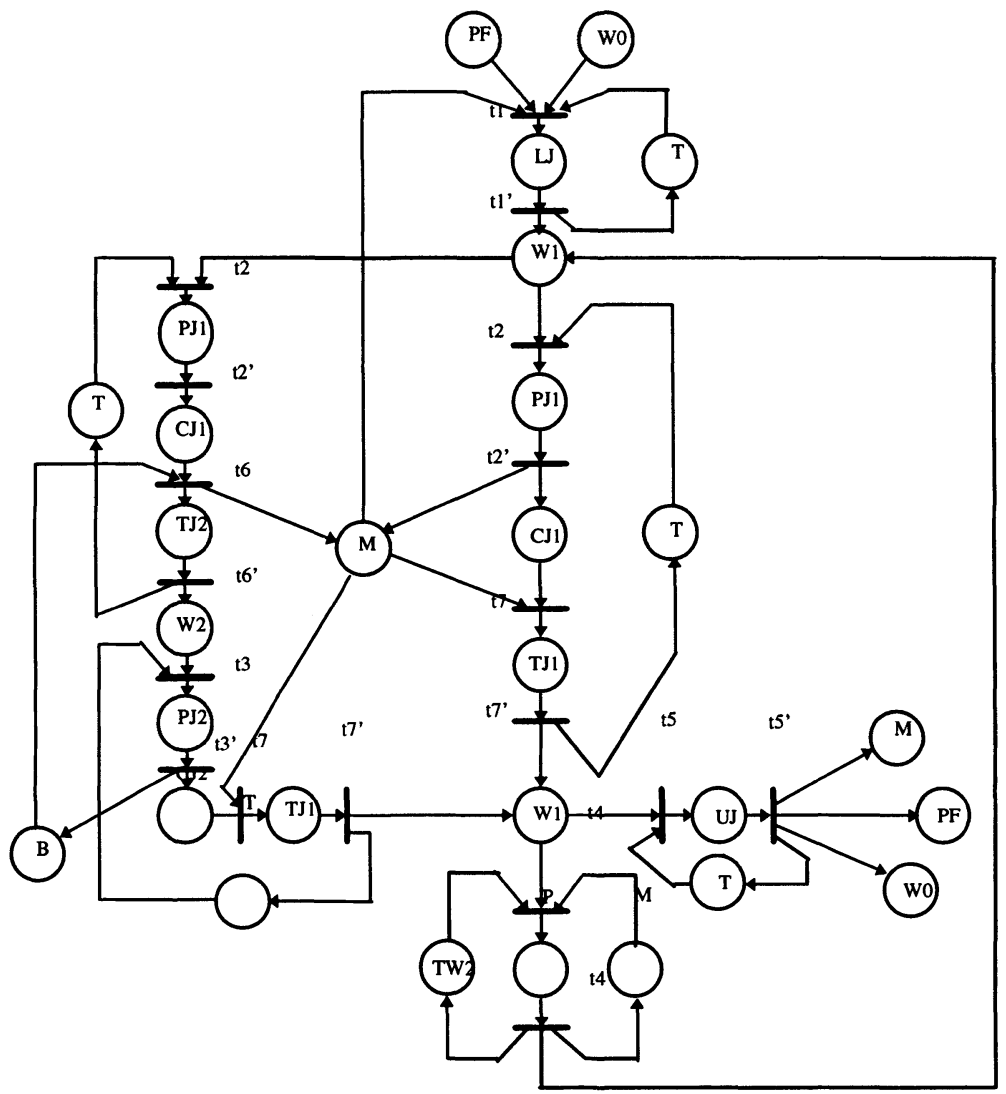

Figure 6 Structured macro Petri-net (SMPN) model for job-flow of FMS for TEST_SYS. 
- for transitions,

$\mathrm{t} 1, \mathrm{t} 1$ ' $\quad$ starting event ( $\mathrm{t} 1)$ and finishing event ( $\mathrm{t} 1^{\prime}$ ) of a job-loading process;

$\mathrm{t} 2, \mathrm{t} 2$ ' starting event and finishing event of a process of picking a pallet from a machine;

$\mathrm{t} 3, \mathrm{t} 3^{\prime} \quad$ starting event and finishing event of a process of picking a pellet from a buffer;

t4, t $^{\prime} \quad$ starting event and finishing event of a job-machining process;

t5, t5 $\quad$ starting event and finishing event of a job-unloading process;

t6, t6 ' starting event and finishing event of a process of sending a pallet to a buffer,

$\mathrm{t} 7, \mathrm{t} 7$, starting event and finishing event of a process of sending a pallet to a machine.

- for places,
LJ, UJ
$\mathrm{PJ} 1, \mathrm{PJ} 2$
processes of loading (LJ) and unloading (UJ) a job;
or from a buffer (PJ2);
$\mathrm{TJ} 1, \mathrm{TJ} 2$ processes of that a conveyer (AGV) sends a pallet to a machine (TJ1) or to a buffer (TJ2);
P process of a job-machining;
W0 status of a job which is outside the system;
W1, W2 statuses of a job which is on a machine (W1) or on a buffer (W2);
$\mathrm{CJ} 1, \mathrm{CJ} 2$ statuses which represent that a conveyer has got a pallet from a machine
(CJ1) or from a buffer (CJ2);
TW2 status of a tool in a local magazine;
PF, T, B, M available statuses of a fixture (PF), a tool (T), a buffer (B), and a machine (M).

For brevity, we do not introduce the tool-flow model in this paper. It can be referred to $\mathrm{Bi}$ (1995) for details. 


\section{MACRO STRUCTURISATION OF PLACES AND RESOURCES}

\subsection{Methodology}

- First, structure resources

- then, structure places

5.2 Structures of Resources describe the statuses of resources.

- S1: Pallet resource

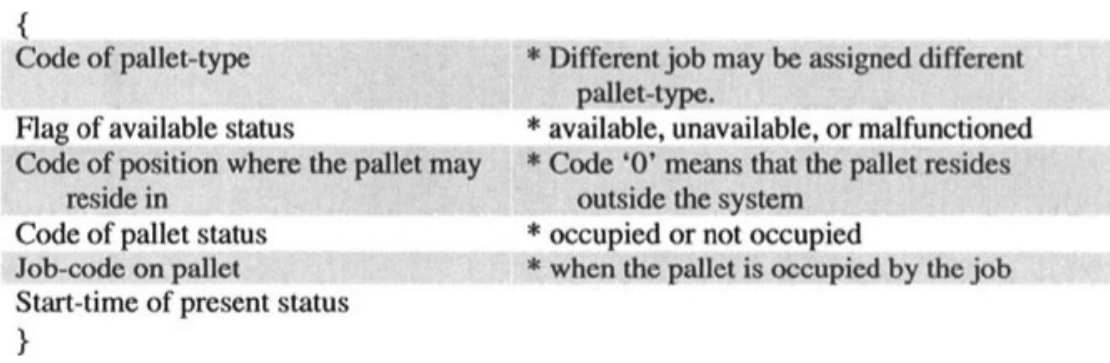

- S2: Position Resource which can be used for Pallet Residing in (PRPR)

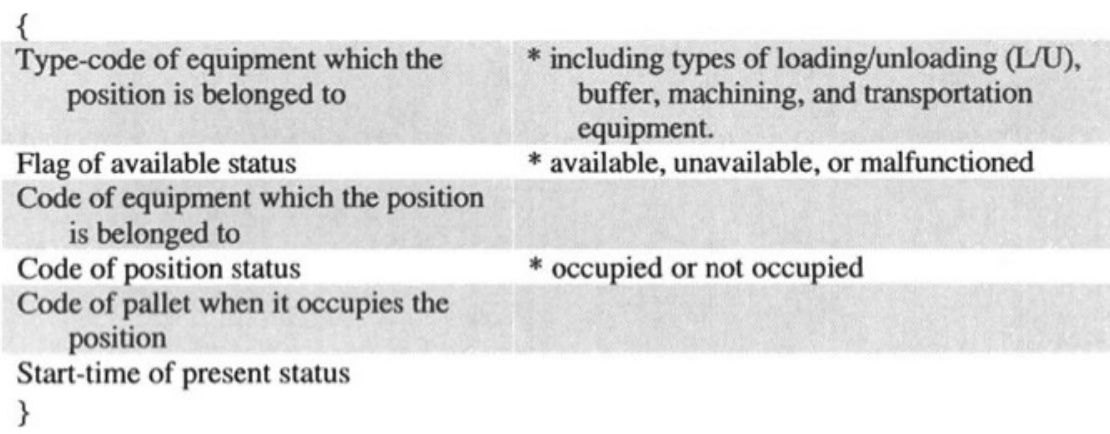

- S3: Tool resource which includes LU tool, transportation tool, and machining tool 
- S31: LU tool resource

$\begin{array}{ll}\begin{array}{l}\text { Code of tool-type } \\ \text { Flag of available status }\end{array} & \begin{array}{c}\text { * Different equipment may be assigned } \\ \text { different type of U/L tool. }\end{array} \\ \begin{array}{l}\text { Code of tool status } \\ \text { Code of equipment which is using the }\end{array} & * \text { available, unavailable, or malfunctioned } \\ \quad \text { tool } & \\ \text { Start-time of present status } & \\ \}\end{array}$

- S32: transportation tool resource

$\begin{array}{ll}\begin{array}{l}\text { Code of tool-type } \\ \text { Flag of available status }\end{array} & \begin{array}{c}\text { Different pallet may require different type of } \\ \text { transportation tool. }\end{array} \\ \begin{array}{l}\text { Code of tool status } \\ \text { Code of pallet which is using the tool }\end{array} & * \text { in use or not in use } \\ \text { Start-time of present status } & \\ \} & \end{array}$

- S33: machining tool resource

\{

Code of tool-type

Flag of available status

Code of tool status

Code of equipment which is using the

tool

Start-time of present status

\}

- S4: Job resource

\{

Code of job-type

Code of finished machining operation

within the machining process

Code of pallet which is used by the job

Start-time of present status

\} 西
* Different job may require different type of machining tool.

* available, unavailable, or malfunctioned

* in use or not in use 
- S5: Workstation resource

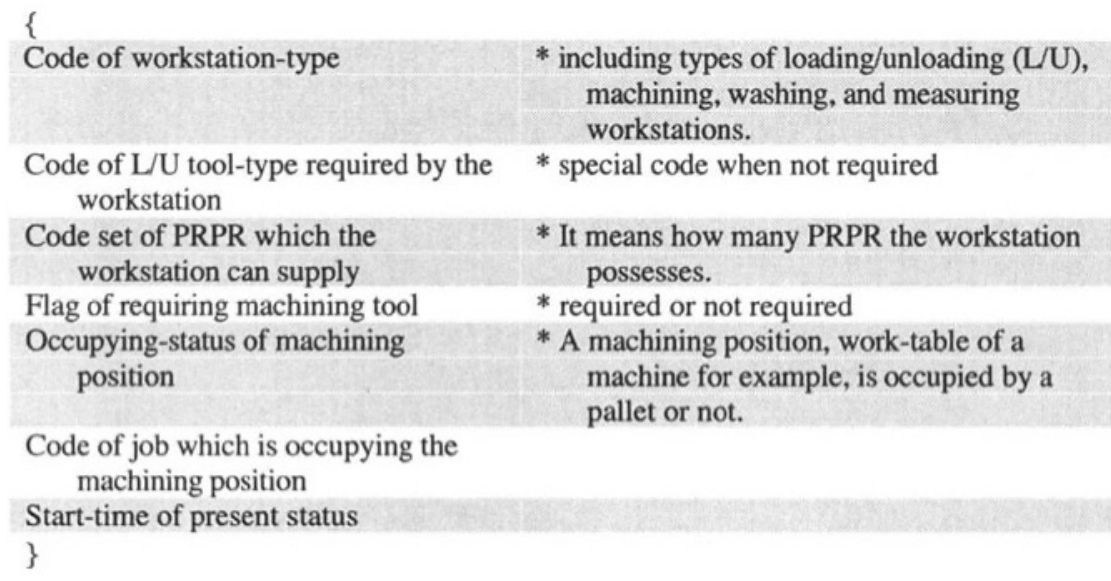

5.3 Structures of Places can be derived from structures of resources.

- Structure PF: $\quad(S 1 \rightarrow$ PF $)$

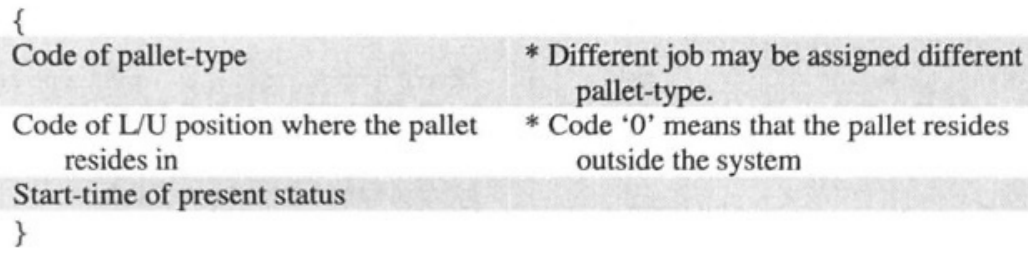

- Structure of W0: (S4 $\rightarrow$ W0)

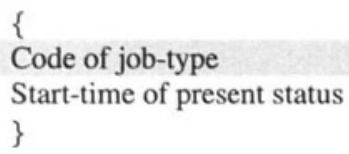

- Structure of M: $\quad(\mathbf{S 2} \rightarrow \mathbf{M})$

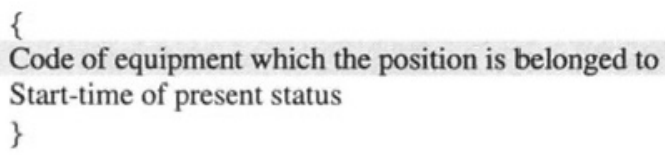


- Structure of T: $\quad(\mathbf{S 3 1} \rightarrow \mathrm{T})$

\{

Code of tool-type

Code of equipment which is using the tool

Start-time of present status \}

- Structure of LJ: (S1, S31, S4, and S5 $\rightarrow$ LJ)

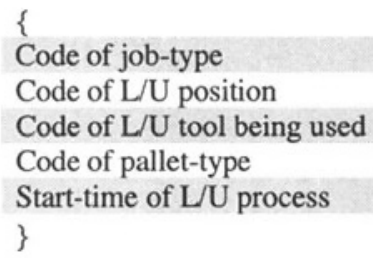

- Structure of PJ1: (S2 and S32 $\rightarrow$ PJ1)

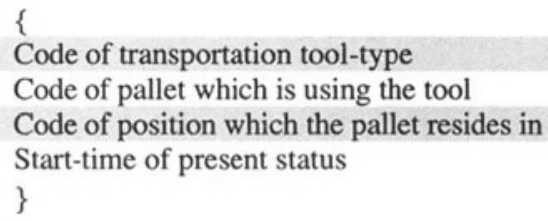

- Structure of W1: $\quad($ S2 $\rightarrow$ W1)

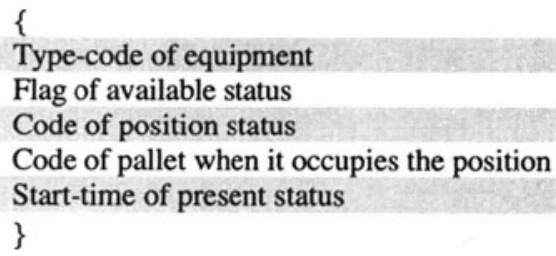

- Structure of CJ1: (S2 and S32 $\rightarrow$ CJ1)

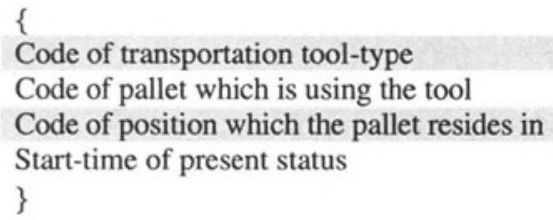




\subsection{Example}

An FMS configuration shown in Figure 7 is composed of:

- $\quad$ a L/U workstation with manual L/U operation;

- a CNC machining workstation possessing two local positions for pallet residing;

- a washer workstation possessing without local position;

- a buffer consisting of four positions for pallet residing;

- $\quad$ an AGV with one position for pallet residing;

- three jobs a, b, and c waiting for entering the system;

- two pallets with one at L/U workstation and the other at one of the four positions of the buffer.

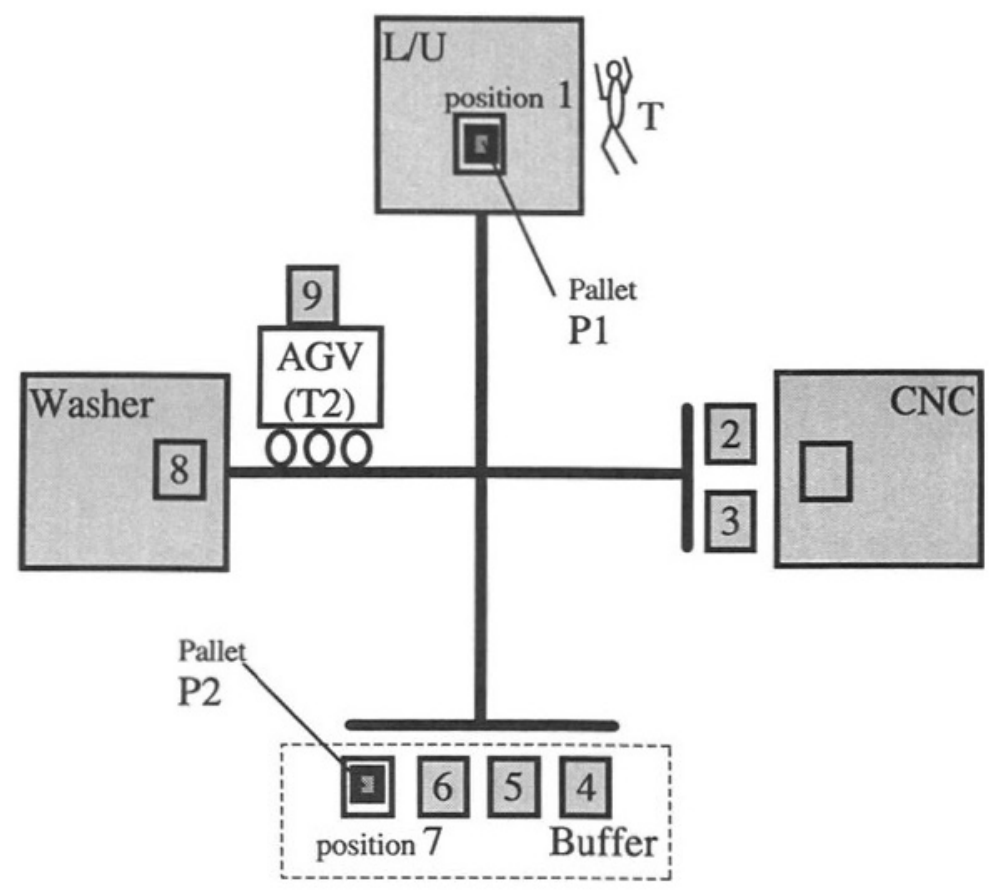

Figure 7 An exemplified example of FMS 


\section{After initialisation}

- $\quad$ Numbers of resource structures, S1, S2, S3 (S31, S32, S33), S4, S5 are

$$
2,9,2(1,1,0), 3,3
$$

- $\quad$ Number of place structures (tokens), PF, W0, M, T, LJ, W1, PJ1, CJ1 derived from resource structures are

t1 firing (start to load job ' $a$ ')

$$
1,3,1,(1,1,0), 0,0,0,0
$$

- Job ' $a$ ' is loading.

- Token numbers in the input places of transition $\mathrm{t1}$ - PF, M, and T are all decreased by 1 and become to be

$$
0,0,(0,1,0) \text {. }
$$

- Token number in the output place $\mathbf{L J}$ increases with 1 and is now equal to 1 .

- The value of the token structure becomes to be

$$
\mathrm{LJ}: \quad\{\mathrm{a}, 1, \mathrm{~T} 1, \mathrm{P} 1, \mathrm{t} 1\}
$$

t1' firing (finish the loading of job ' $a$ ')

- Token numbers in the input places of transition $\mathrm{tl}^{\prime}-\mathrm{LJ}$ is decreased by 1 and become to be 0 now.

- Token numbers in the output places of transition $\mathrm{t1}$ ' - W1 and T both increase with 1 .

- The values of the token structures become to be

W1: $\{\mathrm{L} / \mathrm{U}$ workstation, available, occupied, $\mathrm{P} 1, \mathrm{t} 1$ ' $\}$

$\mathrm{T}: \quad\left\{\mathrm{T} 1, \mathrm{~L} / \mathrm{U}\right.$ workstation, $\left.\mathrm{t} 1^{\prime}\right\}$

t2 firing (transportation tool T2 start to fetch pallet P1 with job 'a' loaded on it)

- Token numbers in the input places of transition $\mathrm{t} 2$ - both $\mathrm{W} 1$ and $\mathrm{T}$ are all decreased by 1 and become to be 0 and $(1,0,0)$ now.

- Token number in the output place of transition $\mathrm{t} 2$ - PJ1 increase with 1 and becomes to be 1 .

- The value of the token structure becomes to be

$$
\text { PJ1: } \quad\{\mathrm{T} 2, \mathrm{P} 1,1, \mathrm{t} 2\}
$$


t2' firing (job ' $a$ ' has been fetched onto the transportation tool)

- Token number in the input place of transition $\mathrm{t}^{2}$ - $\mathrm{PJ} 1$ is decreased by 1 and become to be 0 now.

- Token number in the output place of transition t2' - CJ1 increase with 1 .

- The value of the token structure becomes to be

$\mathrm{CJ} 1: \quad\{\mathrm{T} 2, \mathrm{P} 1,9, \mathrm{t} 2\}$

\section{CONCLUSION}

The SMPN modelling methodology conceived by authors gains the benefits of: (1) the structured places and transitions represent a high level of abstraction; the values of structures of tokens are able to contain more information than that contained by tokens of conventional Petri net method; (2) the SMPN model relates only to the basic events that happen in an FMS, therefore there are no influences to the scale and complexity of the model when the quantity of jobs, devices, buffers, and alternative machining routes of jobs increases. Consequently a succinct SMPN model can be built.

The testing environment created by the authors is now running at its beginning stage. A few FMS controllers have been tested in this environment as case-studies and test-beds for the environment. It is found that the environment is capable of finding faults within the tested FMS controllers covering the following items: (1) unreasonable or false transition commands when simulating both normal situations and resources malfunctions, (2) violation of dispatchingpriority rules, and (3) deadlock in job-flow and tool-flow.

For an FMS controller, one other functionality is the scheduling capability. The authors have not finished the research on how to test the scheduling capability yet. This is what the authors want to do in the next stage.

\section{REFERENCES}

Bi, Z. and Zhu, Y. and Deng, Z. (1995) Theory and Application of SMPN Modelling Methodology and Algorithm for FMS-controller Testing, Scientific report \#20, Nanjing University of Science and Technology.

Deng, Z. and Wang, L. and Liu. X. (1989) A Study of Modelling Part Flow and Tool Flow for FMS, Software for Manufacturing, North-Holland.

Digital Equipment Co. (1990) Manufacturing Enterprise Handbook.

Huang, X. and Deng, Z. (1995) Algorithm for Generation of Testing-case in FMS-controller Testing, Scientific report \#10, Nanjing University of Science and Technology.

Peterson, J. L. (1981) Petri Net Theory and the Modelling of Systems, Prentice-Hall, Inc..

Rembold, U. and Nnaji, B. O. and Storr, A. (1993) Computer Integrated Manufacturing and Engineering, Addison-Wesley.

Schach, S. R. (1990) Software Engineering, Aksen Associate, Inc..

Zhu, Y. and Bi, Z. and Deng, Z. (1995) Testing Mechanism for FMS-controller Testing, Scientific report \#10, Nanjing University of Science and Technology. 